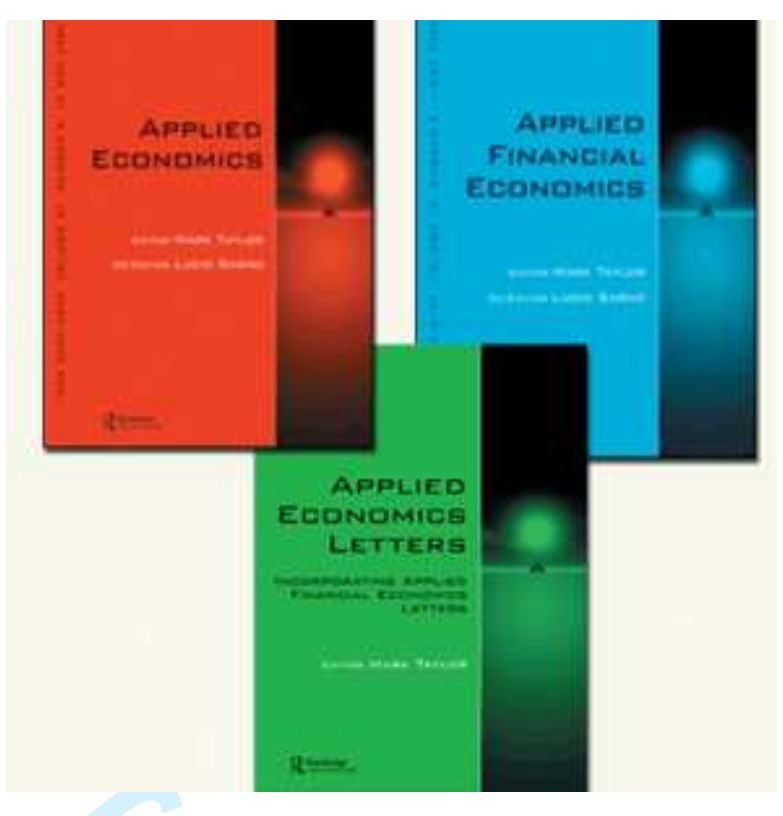

\title{
Small is not that beautiful after all: Measuring the scale efficiency of Tunisian High Schools using a DEA-bootstrap method
}

\begin{tabular}{|c|c|}
\hline Journal: & Applied Economics \\
\hline Manuscript ID: & APE-2011-0073 \\
\hline Journal Selection: & Applied Economics \\
\hline $\begin{array}{r}\text { Date Submitted by the } \\
\text { Author: }\end{array}$ & 17-Feb-2011 \\
\hline Complete List of Authors: & $\begin{array}{l}\text { Essid, Hédi; Institut Supérieur de Gestion } \\
\text { Ouellette, Pierre; Université du Québec à Montréal, Economics } \\
\text { Vigeant, Stephane; Universite des sciences et technologie de Lille, } \\
\text { Institut des sciences economiques et de management }\end{array}$ \\
\hline JEL Code: & $\begin{array}{l}\text { D24 - Production|Capital and Total Factor Productivity|Capacity }< \\
\text { D2 - Production and Organizations < D - Microeconomics, I21 - } \\
\text { Analysis of Education < I2 - Education < I - Health, Education, and } \\
\text { Welfare }\end{array}$ \\
\hline Keywords: & $\begin{array}{l}\text { Returns to scale, Data envelopment analysis, Bootstrap, Quasi-fixed } \\
\text { inputs, Optimal School Size }\end{array}$ \\
\hline
\end{tabular}




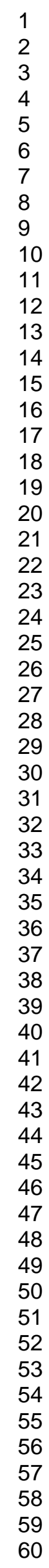

\section{SCHOLARONE ${ }^{m}$ \\ Manuscripts}

7

25

26

27

29

30

31

33

34

35

36

37

38

41

42

43

45

46

47

48

49

51

52

53

54

55

57

58

59

60 


\title{
Small is not that beautiful after all: Measuring the scale efficiency of Tunisian High Schools using a DEA-bootstrap method
}

\author{
Hédi Essid \\ Institut Supérieur de Gestion \\ 41, Rue de la liberté, Cité Bouchoucha 2000 \\ Le Bardo, Tunis-Tunisie \\ e-mail: hedi.essid@ihec.rnu.tn \\ Pierre Ouellette \\ Department of Economics, \\ Université du Québec à Montréal \\ PO Box 8888, Station Centre-Ville \\ Montréal, Québec \\ Canada H3C 3P8 \\ e-mail: ouellette.pierre@uqam.ca \\ and \\ Stéphane Vigeant (corresponding author) \\ IESEG School of management \\ and \\ EQUIPPE (Universités de Lille) \\ Faculté des Sciences Économiques et Sociales \\ Université des Sciences et Technologies de Lille \\ 59655 Villeneuve d'Ascq Cédex, \\ France \\ Phone: +33 (0)3 203363 56, Fax: +33 (0)3 20436655 \\ e-mail: Stephane.Vigeant@univ-lille1.fr
}

February 2011 


\begin{abstract}
Allocation of resources to schools in a centrally managed State system, as the Tunisian one, should depend on the performance of the individual institutions. The optimal size is of crucial importance in this context and we need accurate measurement for sound policies. This paper discusses and implements a nonparametric statistical test procedure for organization scale efficiency. This procedure allows us to test whether the observed scale efficiency is optimal or not using a smooth bootstrap methodology for efficiency measures estimated using DEA methods. Because school principals do not control for the size of their institution, i.e. the capital available at decision time, the scale efficiency measures are defined so as to include quasi-fixed inputs. The results show that scale efficiency measures are subject to sampling variation. We also found that the schools that are scale efficient are usually mid-sized and large schools, when size is measured by the number of students. This contradicts the largely shared view among decision makers that small schools were optimal.
\end{abstract}

Keywords: Returns to scale, Data envelopment analysis, Bootstrap, Quasi-fixed inputs, Tunisia School Efficiency, Optimal School Size. 


\section{Introduction}

Public education systems, like the Tunisian one, face scarce resources that have to be shared between educational institutions. One of the objectives of a well designed educational policy is to allocate these resources to the right number of schools. It implies that identifying the optimal size of the institutions is crucial for the organization of the education system. But this debate raises some arguments by proponents of different views of the system. Smaller institutions are said to be able to create a motivating environment and a comfortable sense of proximity for the students, e.g. Coladarci and Cobb (1996) or Monk (1987), while larger institutions have the advantage of gathering together a large amount of resource and offering an optimal mix of services, e.g. Kuziemko (2006). In fact, arguments can be made for both types of school as it is shown in the literature review by Leithwood and Jantzi (2009).

Costs have been central to the analysis of schools optimal size. Most of the earlier studies focused on concepts of average cost. Morris (1964) shows that the cost per student is the lowest in high school with less than 500 students while Turner and Thrasher (1970) find that cost improvements are very slim beyond 1000 students in the same institution. Monk (1987) shows that beyond 400 students, there are no rooms for economies of scale. These results are consistent with the view that the average cost function of schools must be U-shaped (Fox, 1981). McGuire (1989) finds results consistent with an increasing average cost for schools that reach a certain size (beyond 2,000 students, the unit costs starts to be increasing). More recently, Colgrave and Giles (2005) concluded that the optimal efficient scale for a high school is 1,540 students, while Foreman-Peck and Foreman-Peck (2006) are in the range of 540 students. It is also common to find a negative relationship between the average cost and the size for school with less than 1,000 students (e.g. Stiefel et al., 2009, Chabotar, 1989, and Kumar 1983).

In this paper, we offer an element of response to this debate by addressing the question of the qualitative measurement of returns to scale in organizations. This measure allows us to determine if organizations' activities are characterized by either increasing, decreasing or constant returns to scale. Based on this assessment, we can determine the optimal size of a school. The methodology is applied to a sample of Tunisian high school and we use it to give some insight on the way education policies might be oriented. 
Our approach is anchored in the DEA tradition. Returns to scale have received substantial attention, leading to three measurement methods under the DEA approach: the scale efficiency method (Färe and Grosskopf (1985) and Färe et al. (1994)), the sum of intensity variables method of Banker (1984), Banker and Thrall (1992) and Färe and Grosskopf (1994), and the dual variable-sign-method of Banker et al. (1984). Banker et al. (1994) have shown that these three methods are equivalent. It is not uncommon however, that practitioners using these methods neglect the statistical content of the efficiency measures and do not distinguish between the true value and the estimator.

If the data are generated by a distribution with bounded support, based on the real production set, then the scale efficiency is measured with respect to an estimated frontier with data obtained from an unobserved Data Generating Process (DGP). Under this framework, the observed organization's efficiency is a statistical estimate potentially noisy and spoiled by statistical noises. That is, efficiency measurements are subject to sampling variations. Consequently, it is necessary to adopt a formal statistical methodology that differentiates between the real efficiency value and its estimator in order to evaluate the statistical significance of the results concerning the returns to scale of an organization. This is what the procedure developed by Simar and Wilson (2002) offers. Their method consists in smoothing the probability distribution of the efficiency scores with a convolution kernel. Then the authors use a reflection method (Silverman, 1986 and Schuster, 1985) to correct the estimator of the probability density in the neighborhood of the boundaries. However, Essid et al. (2010b) show that this test procedure rests on a statistic that can assume infeasible values. That is, the test is defined as the ratio of two efficiency scores, those being measured under two different assumptions on the return to scale. Then, in the bootstrap simulations, the efficiency scores are generated from two different DGPs. This might ultimately lead to values of the score that are not admissible: The scale efficiency pseudo-scores may end up taking values above one and this is clearly not allowed, by definition.

Essid et al. (2010b) have proposed a solution to this drawback of an otherwise working procedure. They have developed a smooth bootstrap method that ensures that the pseudo-scores of the efficiency scale assume only admissible values and they base the test procedure on a consistent DGP of the scale efficiencies. Furthermore, instead of considering a global test for returns to scale, a test specific to each decision making unit (DMU) is implemented. 
The methodology used in this paper to measure and test the scale efficiency of Tunisian high school is based on Essid et al. (2010b). We have adapted this method to take into account the fact the school principals do not control the physical size of their institution. This means that we include quasi-fixed inputs (also known as non-discretionary inputs) in the DEA model as it was done in Banker and Morey (1986).

The Tunisian high school network offers a perfect study case. Firstly, the education system is state run and resource allocation is centralized. The Department of education creates and implements the programs, it hires the teachers and administrative staff and dispatch them based on the estimated needs of the schools and finally it allocates the operating budget between the different institutions. (The share of the money from the private sector is minimal.) This is to ensure that all schools have more or less the same resources per student. Secondly, the size of the schools measured by the number of students varies a lot over the country. This offers a perfect environment to study the correlation between the size of schools and their returns to scale characterizations. Thirdly, policy makers in the Tunisian Department of Education strongly believe that smaller the institution is, better the performance will be. This raises the problem of the optimal size of the institution and of the scale characterization that schools need to have to profit from the scale efficiency.

This paper offers a test of the scale efficiency of high schools in Tunisia and we check on the claim made by the department of education decision makers that smaller schools are required. We also check the robustness of our results with respect to the simulation methods used. This is done by comparing the results obtained under a homogenous bootstrap procedure to those obtained using a heterogeneous bootstrap procedure.

\section{Production technology and returns to scale}

Consider a production activity that uses two sets of inputs, one containing those under the direct control of the decision maker, $x=\left\{x_{i_{1}}, i_{1}=1, \ldots, m_{1}\right\}$, and one containing those not under her control at decision time, $z=\left\{z_{i_{2}}, i_{2}=1, \ldots, m_{2}\right\}$, to produce the output vector, $y=\left\{y_{r}, r=1, \ldots, s\right\}$. The production possibility set of this activity is defined as:

$\Psi=\left\{(x, z, y) \in \mathbb{R}_{+}^{m_{1}+m_{2}+s} \mid(x, z, y)\right.$ is feasible $\}$. 
We suppose that the production set $\Psi$ is closed, satisfies free disposal of inputs, is bounded for finite inputs and positive outputs require positive inputs. Except for our inclusion of quasi-fixed inputs in the production process, these are standard assumptions on the technology and are discussed in Färe (1988), among others.

To characterize the returns to scale environment of the firms and to test for their type, specific production sets are required. The returns to scale are characterized by the way one can either expand the scale in the production set or shrink it or both. That is, the technology exhibits non increasing (nirs), non decreasing (ndrs) or constant (crs) returns to scale depending on the value assumed by the positive scalar $\alpha$ in the following set:

$\Psi^{k}=\left\{(x, z, y) \in \Psi \mid(\alpha x, \alpha z, \alpha y) \in \Psi\right.$ for all $\left.\alpha \in \mathrm{K}^{k}\right\}$, for $k=$ nirs, ndrs, crs

where $\mathrm{K}^{\text {nirs }}=[0,1), \mathrm{K}^{\text {ndrs }}=[1, \infty)$ and $\mathrm{K}^{\text {crs }}=[0, \infty)$. A technology that exhibits ndrs, nirs or crs in different regions of the production frontier is said to be characterized by variable returns to scale (vrs). This production possibility set is denoted $\Psi^{v r s}$.

It is possible to define an input oriented technical efficiency measure in the sense of Farrell (1957) with respect to the various assumptions concerning the returns to scale. That is:

$\theta^{k}(x, z, y)=\min \left\{\theta \mid(\theta x, z, y) \in \Psi^{k}\right\}$, where $k=$ nirs, ndrs, crs and vrs.

The scalar $\theta(x, z, y)$ is a radial measure of efficiency that gives the maximal factor by which we can reduce $x$ so that $y$ can still be produced, given the quasi-fixed input vector $z$.

From Färe and Grosskopf (1985), the (technical) efficiency measures defined in (3) can be used to construct scale efficiency measures for each organization. A scale efficiency measure is the ratio of the efficiency measure under crs technology and a vrs type technology. That is:

$$
S_{1}(x, z, y)=\frac{\theta^{\text {crs }}(x, z, y)}{\theta^{\text {vrs }}(x, z, y)} \leq 1 .
$$

We say that the production technology is of the crs type if $S_{1}(x, z, y)=1$. To determine the returns to scale of the technology when $S_{1}(x, z, y)<1$ we compute a second ratio that is less restrictive than the initial ratio. That is: 
$S_{2}(x, z, y)=\frac{\theta^{\text {nirs }}(x, z, y)}{\theta^{\text {vrs }}(x, z, y)} \leq 1$.

When $S_{2}(x, z, y)=1$ the technology is said to exhibit decreasing returns to scale and for values strictly less than one, i.e. $S_{2}(x, z, y)<1$, it is said to exhibit increasing returns to scale.

\section{Efficiency estimation and statistical model}

The description of the technology presented in the previous section characterizes the true unobserved model. This means that the frontier and the scale efficiency measures $S_{1}(x, z, y)$ and $S_{2}(x, z, y)$ are not observed. These quantities must be estimated using data.

Consider a sample of $n$ observations, $\Psi_{0}=\left\{\left(x_{j}, z_{j}, y_{j}\right)\right\}_{j=1}^{n}$. Since the true production set $\Psi$ is not observed, the efficiency score, defined as the gap between the frontier of that set and the units observed performance, is not available. Consequently, the best we can do is to estimate it from the sample, $\Psi_{0}$, by estimating the "missing" frontier of the production set. To do this, we use the convex hull of the sample. The smallest convex envelop of the data gives the DEA estimator in the vrs case. That is:

$$
\hat{\Psi}^{v r s}=\left\{(x, z, y) \in \mathbb{R}^{m_{1}+m_{2}+s} \mid x \geq \sum_{j=1}^{j=n} \lambda_{j} x_{j}, z \geq \sum_{j=1}^{j=n} \lambda_{j} z_{j}, y \leq \sum_{j=1}^{j=n} \lambda_{j} y_{j}, \sum_{j=1}^{j=n} \lambda_{j}=1 \forall j=1, \ldots, n\right\} .
$$

To obtain the estimators for the other types of returns to scale, $\hat{\Psi}^{\text {nirs }}, \hat{\Psi}^{\text {ndrs }}$ and $\hat{\Psi}^{\text {crs }}$ it is sufficient to slightly alter the constraint on the sum of the $\lambda_{j}$. That is, in the nirs case we have $\sum_{j=1}^{j=n} \lambda_{j} \leq 1$, and in the $n d r s \sum_{j=1}^{j=n} \lambda_{j} \geq 1$ must hold and finally, to obtain a crs envelop, no constraints on the $\lambda_{j}$ are necessary other than non negativity of the $\lambda \mathrm{s}$.

Farrell's efficiency measures are obtained by substituting $\hat{\Psi}^{k}$ to $\Psi^{k}$ in equations (3). This gives:

$$
\hat{\theta}^{k}(x, z, y)=\min \left\{\theta \mid(\theta x, z, y) \in \hat{\Psi}^{k}\right\} k=v r s, \text { nirs, ndrs, crs }
$$


The resulting scale ratio estimators are $\hat{S}_{1}=\hat{\theta}^{\text {crs }}(x, z, y) / \hat{\theta}^{\text {vrs }}(x, z, y)$ and $\hat{S}_{2}(x, z, y)=\hat{\theta}^{\text {nirs }}(x, z, y) / \hat{\theta}^{v r s}(x, z, y)$

To insure the consistency of the estimator, it is necessary to have an explicit understanding of the way the data are generated. In other words, we have to specify a statistical model that allows us for a full to characterization of the data generating process (DGP). This is the content of the following assumption.

Assumption A1: The set of observations $\left\{\left(x_{j}, z_{j}, y_{j}\right)\right\}_{j=1}^{n}$ are identically and independently distributed (i.i.d.) random variables with probability density function $f(x, z, y)$ defined on $\Psi$.

Because Farrell's efficiency measure is radial and because the vector of input $x$ has an equivalent polar representation to the usual Cartesian representation, we have three equivalent representation of the input-output combination. That is:

$(x, z, y) \Leftrightarrow(\omega, \eta, z, y) \Leftrightarrow(\theta, \eta, z, y)$

where $\omega(x)=\|x\|=\sqrt{x^{T} x}, \eta=\left(\eta_{1}, \ldots \eta_{i_{1}}, \ldots, \eta_{m_{1}-1}\right)$ and $\eta=\eta(x) \in[0, \pi / 2]^{m_{1}-1}$ is the angle. Using the law of conditional probability, the joint probability density of $(\theta, \eta, z, y)$ can be decomposed to obtain:

$f(\theta, \eta, z, y)=f(\theta \mid \eta, z, y) f(\eta \mid z, y) f(z \mid y) f(y)$

Assumption A2: The probability density function $f(x, z, y)$ is continuous on the interior of $\Psi$, and $f\left(x^{\partial}(z, y), z, y\right)>0$ where $x^{\partial}(z, y)=\theta(x, z, y) x$ is any point on the frontier of $\Psi$.

Note that points that are not feasible, i.e. the points not in $\Psi$, have zero probability and the density is identically equal to zero.

Assumption A3: The efficiency measure $\theta(x, z, y)$ is differentiable in $x, y$, and $z$.

This assumption gives the necessary smoothness of the frontier function. Kneip et al. (1998) have shown that the convergence rate of the estimator depends on this smoothing condition. However, the smoothing condition we have used is slightly stronger than the one 
required for convergence. As shown by Kneip et al. (1998), it is sufficient that the efficiency measure satisfy a Lipschitz condition.

Assumption A1, A2 and A3 together define the statistical model that allows us to characterize the DGP, denoted $\mathfrak{I}$. In fact, the DGP is entirely characterized by the production possibility set $\Psi$ and the density $f$. That is, $\mathfrak{I}=(\Psi, f)$.

This statistical model ensures the convergence of the estimator and permits a characterization of the asymptotic properties of nonparametric estimators of the DEA type. In particular, Kneip et al. (1998) have shown the consistency of the DEA estimator in the multidimensional case. They show that for a vrs technology with $m$ inputs, the rate of convergence is of order $n^{-2 / m+s+1}$ where $n$ is the number of DMU. Park et al. (2010) show that for a crs technology, the convergence rate of the DEA estimator is faster, as it is of the order $n^{-2 / m+s}$. These results do not make the difference between discretionary and non discretionary inputs. However, Essid et al. (2010c) show that, as long as the efficiency measure is radial, the convergence of the estimator is kept independently of the returns to scale the technology exhibits. An important point made is that since it is a nonparametric estimator, the convergence rate is subject to the curse of dimensionality, and the rate of convergence decrease rapidly with the number of quasi-fixed inputs. Bootstrap simulation procedures are used to construct confidence intervals, to correct the estimator's bias and to test statistical hypothesis, as it is now the norm.

\section{Hypothesis testing and returns to scale}

The traditional approach to qualitative returns to scale measures starts from Färe and Grosskopf (1985). The central argument of the approach is that the ratio of efficiency measures calculated under different returns to scale assumptions, $\hat{S}_{1 j}=\hat{\theta}^{\text {crs }}\left(x_{j}, z_{j}, y_{j}\right) / \hat{\theta}^{\text {vrs }}\left(x_{j}, z_{j}, y_{j}\right) \leq 1$, reveal some information on the nature of the returns to scale of the DMU. When $\hat{S}_{1 j}=1$, the triple $\left(x_{j}, z_{j}, y_{j}\right)$ or its projection on the estimated frontier belong to a technology characterized by constant returns to scale (crs-technology). When $\hat{S}_{1 j}<1$, the observation or its projection is not on a vertex or a side where the technology is crs. When returns to scale are not constant, this approach can be extended to determine the exact nature of the returns to scale, as we will see below. 
Obviously, the scale ratio is computed using an estimated frontier, not the true one. Suppose that for an observation, say $\left(x_{j}, z_{j}, y_{j}\right)$, we obtain $\hat{S}_{1 j}<1$, then without a formal statistical test procedure it is impossible to identify if this is a true scale inefficiency or the result of sampling variations. That is, since the scale ratio is calculated based on an estimated frontier, a score strictly smaller than one can be attributed to sampling variations. Consequently, we have to test the hypothesis that $S_{1 j}\left(x_{j}, z_{j}, y_{j}\right)=1$ using the statistic $\hat{S}_{1 j}\left(x_{j}, z_{j}, y_{j}\right)$ to determine if the DMU is really scale inefficient.

The test procedure is in two steps. The first step consists in testing the null that a given triple $(x, z, y)$ is scale efficient, i.e. its technology is of the crs type. The alternative hypothesis has to be less restrictive. One natural hypothesis is that the triple $(x, z, y)$ is characterized by a vrs-technology. Then we have:

$$
\begin{array}{r}
\text { Test\#1: } H_{0}: S_{1}(x, z, y)=1 \\
H_{A}: S_{1}(x, z, y)<1
\end{array}
$$

If the hypothesis $H_{0}$ is rejected, we still have to identify whether the returns to scale are increasing or decreasing. This will work if we can find a "new" null hypothesis that is less restrictive than the one in the first test. One way of doing this is to suppose that under the null hypothesis the triple $(x, z, y)$ is subject to decreasing returns to scale $(d r s)$. Then, the alternative hypothesis would be that the triple $(x, z, y)$ is subject to an increasing return to scale (irs) technology, since constant returns to scale have already been rejected in the first test. Thus, the second test is:

$$
\begin{array}{r}
\text { Test \#2: } H_{0}^{\prime}: S_{2}(x, z, y)=1 \\
H_{A}^{\prime}: S_{2}(x, z, y)<1
\end{array}
$$

The test statistics for the first and second test are $\hat{S}_{1}(x, z, y)=\hat{\theta}^{\text {crs }}(x, z, y) / \hat{\theta}^{\text {vrs }}(x, z, y)$ and $\hat{S}_{2}(x, z, y)=\hat{\theta}^{n i r s}(x, z, y) / \hat{\theta}^{\text {vrs }}(x, z, y)$, respectively. Given a critical value $c_{\alpha}>0$ for a given 
test size, $\alpha$, the decision rules are such that we reject $H_{0}$ each time $\left(1-\hat{S}_{1}(x, z, y)\right) \geq c_{\alpha}$ in the first case and each time $\left(1-\hat{S}_{2}(x, z, y)\right) \geq c_{\alpha}$ in the second case.

This test procedure is applied to each observation $\left(x_{j}, z_{j}, y_{j}\right)$ in $\Psi_{0}$. Consequently, we have at least $n$ identical hypothesis testing procedure to run, leading to $n$ decisions concerning the returns to scale for each of the $n$ individual organizations represented in $\Psi_{0}$.

To apply these tests we need to either choose the critical value $c_{\alpha}$ for a given test size or alternatively we can estimate the $p$-value for each test. In both cases, it is necessary to have the distribution of the test statistics. This is the problem discussed in the next section.

\section{Bootstrapped test statistics}

To determine the critical values and the decision rules, we use the approach developed in Essid, et al. (2010b), which is based on Simar and Wilson (2002). This approach allows us to find an approximation of the sampling distribution of the estimators of both scale efficiency ratios, $\hat{S}_{1}$ and $\hat{S}_{2}$. This approximation rests on the bootstrap method that consists in identically replicating the empirical DGP many times and study the behavior this set of bootstrapped estimates. To implement the procedure, we first generate, from the original sample $\Psi_{0}, B$ pseudo-samples: $\Psi_{b}^{*}, b=1, \ldots, B$. Then, the original estimation method (DEA in our case) is applied to each pseudo-samples to obtain the bootstrap estimator of the test statistic $\hat{S}_{1 b}^{*}$ for $\hat{S}_{1}$ (and $\hat{S}_{2 b}^{*}$ for $\hat{S}_{2}$ ). This procedure allows us to estimate the empirical distribution of $\left(\hat{S}_{1}^{*}-\hat{S}_{1}\right)$ (and of course $\left(\hat{S}_{2}^{*}-\hat{S}_{2}\right)$, used to approximate the unknown distribution of the statistic $\left(\hat{S}_{1}-S_{1}\right)$ (and $\left.\left(\hat{S}_{2}-S_{2}\right)\right)$, under the null hypothesis.

The bootstrap relies on the principle that the pseudo-samples $\Psi_{b}^{*}$ are generated so that:

$$
\left(\hat{S}_{1}-1\right)\left|\mathfrak{J}^{\text {crs }} \stackrel{\text { approx }}{\sim}\left(\hat{S}_{1}^{*}-\hat{S}_{1}\right)\right| \hat{\mathfrak{I}}^{\text {crs }} \text { for Test \# } 1
$$

and 
$\left(\hat{S}_{2}-1\right)\left|\mathfrak{J}^{\text {nirs }}{ }^{\text {approx }}\left(\hat{S}_{2}^{*}-\hat{S}_{2}\right)\right| \hat{\mathfrak{I}}^{\text {nirs }}$ for Test \# 2,

where $\hat{\mathfrak{I}}$ is a consistent estimator of the DGP $\mathfrak{I}$ under the hull hypothesis, i.e. crs in the first case and nirs in the second case.

To generate the pseudo-efficiencies $\hat{\theta}^{* r s}, \hat{\theta}^{* v r s}$ and $\hat{\theta}^{* n i r s}$, and then the statistics $\hat{S}_{1}^{*}$ and $\hat{S}_{2}^{*}$, we first use a homogenous bootstrap methodology developed by Simar and Wilson (1998). This procedure rests on the assumption that the efficiency structure is homogenous. That is, the efficiency score $\theta$ is independent of $(\eta, z, y): f(\theta \mid \eta, z, y)=f(\theta)$. A consistent estimator of $f$, obtained using a kernel estimator and corrected by Schuster's (1985) and Silverman (1985) is defined as follows:

$$
\hat{f}^{c}(t)=\left\{\begin{array}{c}
2 \hat{g}(t) \text { if } t \leq 1 \\
0 \quad \text { otherwise }
\end{array}, \text { where } \hat{g}(t)=\frac{1}{2 n h} \sum_{j=1}^{j=n}\left[\phi\left(\frac{t-\hat{\theta}_{j}}{h}\right)+\phi\left(\frac{t-2+\hat{\theta}_{j}}{h}\right)\right] .\right.
$$

We use a normal Gaussian kernel, denoted $\phi$, and the bandwidth, $h$, is set following the normal reference rule (Silverman (1986)).

The robustness of the testing procedure with respect to the simulations is checked by relaxing the homogeneity assumption in a second simulation experiment. We run a smooth heterogeneous bootstrap simulation, based on a methodology developed in Simar and Wilson (2000). Then the test procedure is re-run with the new bootstrapped dataset. In this case, the density $f$ is estimated by: ${ }^{1}$

$$
\hat{f}^{c}(u)=\left\{\begin{array}{l}
2 \hat{f}(u) \text { if } u \in \Omega \\
0 \text { otherwise }
\end{array},\right.
$$

where $\hat{f}(u)=\frac{1}{2 n h^{m_{1}+m_{2}+s}} \sum_{j=1}^{j=n}\left[K_{1}\left(\frac{u-u_{j}}{h}\right)+K_{2}\left(\frac{u-u_{R j}}{h}\right)\right], K_{l}(\bullet)$ is the probability density of a normal vector with zero mean and variance-covariance matrix $\hat{\Sigma}_{l}, l=1,2, u_{j}=\left(\theta_{j}, \eta_{j}, z_{j}, y_{j}\right)$,

\footnotetext{
${ }^{1}$ Both simulation procedures are presented in Essid et al. (2010a).
} 
and $u_{R j}=\left(2-\theta_{j}, \eta_{j}, z_{j}, y_{j}\right)$. The bandwidth parameter is set according to Silverman's normal rule.

The pseudo-scores are generated in three steps. We begin by presenting the procedure for the first test:

Step 1: Generate $\hat{\theta}^{*}$ from $\hat{\theta}^{\text {crs }}$.

Step 2: Generate the pseudo-sample $\Psi^{*}=\left\{\left(x^{*}, z^{*}, y^{*}\right)\right\}$ using the estimator $\hat{f}^{c}$ of $f$ given by equation (12), in the case of the homogenous bootstrap and using equation (13) in the case of the heterogeneous bootstrap.

Step 3: Compute $\hat{\theta}^{* r s}$ and $\hat{\theta}^{*}$ rrs using the same pseudo sample $\Psi^{*}$ for both measures, insuring that the pseudo-score $\hat{S}_{1}^{*}=\hat{\theta}^{* c r s} / \hat{\theta}^{* v r s}$ has an admissible value, i.e. $\hat{S}_{1}^{*}=\hat{\theta}^{* r s} / \hat{\theta}^{* v r s} \leq 1{ }^{2}$

This three step procedure is then replicated $B$ times to obtain the statistic $\hat{S}_{1 j}=\hat{\theta}^{\text {crs }}\left(x_{j}, z_{j}, y_{j}\right) / \hat{\theta}^{v r s}\left(x_{j}, z_{j}, y_{j}\right)$ for each triple $\left(x_{j}, z_{j}, y_{j}\right)$ in the dataset $\Psi_{0}$. Then we obtain $B$ pseudo-values $\hat{S}_{1 j b}^{*}=\hat{\theta}_{b}^{* r r s}\left(x_{j}, z_{j}, y_{j}\right) / \hat{\theta}_{b}^{*}$ vrs $\left(x_{j}, z_{j}, y_{j}\right), b=1, \ldots, B$ for all $j=1, \ldots, n$.

To obtain the pseudo-scores $\hat{S}_{2}^{*}=\hat{\theta}^{* n i r s} / \hat{\theta}^{* \text { vrs }}$, we only need to bootstrap $\hat{\theta}^{\text {nirs }}$ instead of $\hat{\theta}^{c r s}$ in Step 1 above to recover the pseudo-sample $\Psi^{*}$ used to compute the pseudo-efficiencies. Then, the test statistic $\hat{S}_{2 j}=\hat{\theta}^{n i r s}\left(x_{j}, z_{j}, y_{j}\right) / \hat{\theta}^{\text {vrs }}\left(x_{j}, z_{j}, y_{j}\right)$ is replicated $B$ times, i.e. $\hat{S}_{2 j b}^{*}=\hat{\theta}_{b}^{* n i r s}\left(x_{j}, z_{j}, y_{j}\right) / \hat{\theta}_{b}^{*} r r s\left(x_{j}, z_{j}, y_{j}\right), b=1, \ldots, B$, for each triple in $\Psi_{0}$.

To obtain the critical values and infer the decision rules of both tests, we use the bootstrap analog of the true size of the test, denoted $\alpha$ (i.e. the probability of Type 1 error). For the first and second tests they are respectively defined as $\operatorname{Pr}\left(\hat{S}_{1}^{*} \leq \hat{S}_{1}-c_{\alpha}^{*} \mid H_{0}, \hat{\mathfrak{J}}^{\text {crs }}\right)=\alpha$ and $\operatorname{Pr}\left(\hat{S}_{2}^{*} \leq \hat{S}_{2}-c_{\alpha}^{*} \mid H_{0}, \hat{\mathfrak{J}}^{\text {nirs }}\right)=\alpha$, where $c_{\alpha}^{*}$ is an approximation of the critical value $c_{\alpha}$.

\footnotetext{
${ }^{2}$ This at this stage that we make sure that the DGP is consistent with the hypothesis tested. With this procedure, the test statistic is always less than or equal to one because both efficiency measures are calculated from data coming from the same DGP.
} 
To obtain these critical values, we start by sorting in ascending order the pseudo-scores $\left(\hat{S}_{1 b}^{*}-\hat{S}_{1}\right)\left(\right.$ or $\left.\left(\hat{S}_{2 b}^{*}-\hat{S}_{2}\right)\right)$ for $b=1, \ldots, B$. Then, we eliminate $(1-\alpha) \times 100$ percent of the values at the right end of the ordered sequence of bootstrapped statistics. The critical value $-c_{\alpha}^{*}$ is then the right end of this truncated sequence. Thus, the decision rule is the following: reject the null hypothesis if, for a given size $\alpha, \hat{S}_{1} \leq 1-c_{\alpha}^{*}$ for the first test and $\hat{S}_{2} \leq 1-c_{\alpha}^{*}$ for the second.

Hypothesis tests can be more convincingly used by giving the marginal probability of rejections, or $p$-value. The bootstrap analog of the $p$-value for $H_{0}$ is $\hat{p}=\operatorname{Pr}\left(\hat{S}_{1}^{*} \leq \hat{S}_{1} \mid H_{0}, \hat{\mathfrak{J}}^{\text {crs }}\right)$ for the first test and $\hat{p}=\operatorname{Pr}\left(\hat{S}_{2}^{*} \leq \hat{S}_{2} \mid H_{0}, \hat{\mathfrak{J}}^{\text {nirs }}\right)$ for the second. For each test, we reject null hypothesis when $\hat{p} \leq \alpha$, for a given size $\alpha$.

\section{Application to Tunisian high schools}

The report of the World Bank (2008) on the state of education on the Middle East and North Africa (MENA) concluded that the education system does not satisfy the needs of the society and does not meet the growing ambitions of the people. It is said that the failure to achieve positive results rests mostly on a misallocation of the resources devoted to the education system and to schools operating inefficiently. In the case of Tunisia, Essid et al. (2010a) show that high schools would be able to deliver the same amount of services with $12 \%$ less resources on average. Other than the internal working of institutions, the overall organization of the education system has been under scrutiny.

To improve the internal performance of the education system, the department of education has made a priority of reducing the size of the schools. Table 1 shows that the number of schools and the number of teachers have increased during the period 2001-2005 to keep up with the constant increase in enrolment. It is noteworthy that the number of schools has increased sufficiently rapidly that the average size of the schools, measured by the number of students, has decreased over the same period: from 965 in 2001/2002 it fell to 946 in 2002/2003 and to 911 in 2004/2005. That is, the average size of the Tunisian secondary education establishments has continuously decreased since 2001. This is consistent with the belief of the Tunisian department of education that smaller units are performing better. This clearly raises the question concerning 
the scale characterization of schools and how the optimal size must be determined so that the schools operate at their optimal scale.

Table 1: Evolution of the number of schools and their average size

\begin{tabular}{|c|c|c|c|c|c|}
\hline $\begin{array}{c}\text { Academic } \\
\text { Year }\end{array}$ & $\begin{array}{c}\text { Number of } \\
\text { Schools }\end{array}$ & Enrolment & $\begin{array}{c}\text { Number of student- } \\
\text { class }\end{array}$ & $\begin{array}{c}\text { Number of } \\
\text { Teachers }\end{array}$ & $\begin{array}{c}\text { Average School } \\
\text { Size }\end{array}$ \\
\hline $01 / 02$ & 1,065 & $1,027,812$ & 31,146 & 49,331 & 965 \\
$02 / 03$ & 1,117 & $1,057,233$ & 32,300 & 51,738 & 946 \\
$03 / 04$ & 1,161 & $1,076,238$ & 33,103 & 55,717 & 927 \\
$04 / 05$ & 1,191 & $1,084,878$ & 33,811 & 59,132 & 911 \\
\hline
\end{tabular}

Source: «Bureau des études, de la planification et de la programmation », Department of Education, Tunisia

Here we would like to characterize the returns to scale and test whether the government is right about the optimal size of schools. In order to do this, we apply our bootstrap methodology to a sample of 332 Tunisian high schools. (This sample contains roughly $28 \%$ of the schools for the reference year 2003/2004.). The Bureau des études, de la planification et de la programmation of the Tunisian department of education has provided data for each school for the academic year 2003/2004. The data include total enrolment, the number of students graduating (successfully completing the baccalauréat), the number of teachers and non-teaching staffs, the number of students in residence (accommodation provided by the institution), the number of general teaching and specialized classrooms, and the operating budget. We have used price indices from the Tunisian National Institute of Statistics (consumer price index for 2004). Using these data, we have constructed our output measures and the quantities of quasi-fixed and variable inputs for each high school. ${ }^{3}$

Tunisian high schools supply two types of services: education and residence (full-board accommodation). We use the number of beds (BEDS) and the number of meals served (MEALS) to measure the residence services. The education services are measured using total enrolment (STUDENTS) and the baccalauréat ${ }^{4}$ results (RBAC) which corresponds to the number of successful students per school.

To produce "students", high schools use variable and quasi-fixed inputs. The variable inputs are the total number of teachers (TEACHERS), the number of administrative personnel $(\mathrm{ADM})$, the number of blue collars, essentially maintenance and cleaning staff (BLUECOL), and

\footnotetext{
${ }^{3}$ For details on the data construction the reader is referred to Essid et al. (2010a).

${ }^{4}$ The baccalauréat is the diploma obtained at the end of high school; it is the equivalent of a high school diploma
} 
finally from the operating budget and the consumer price index, we construct a proxy for food and material supplies (F\&MAT). The quasi-fixed factors are given by the number of general classrooms (GROOM) and the number of specialized classrooms (SROOM). Descriptive statistics of the data are given in Table 2.

Table 2: Descriptive Statistics

\begin{tabular}{|c|c|c|c|c|c|}
\hline & Variable & Mean & $\begin{array}{c}\text { Standard } \\
\text { error }\end{array}$ & Minimum & Maximum \\
\hline \multirow{4}{*}{ Outputs } & STUDENTS & 1292,25 & 461,64 & 346 & 2769 \\
\hline & BEDS & 123,65 & 188,31 & 0 & 931 \\
\hline & MEALS & 173,35 & 220,00 & 0 & 931 \\
\hline & RBAC & 180,66 & 94,05 & 18 & 526 \\
\hline \multirow{4}{*}{$\begin{array}{l}\text { Variable } \\
\text { inputs }\end{array}$} & TEACHERS & 72,53 & 24,32 & 26 & 152 \\
\hline & $\mathrm{ADM}$ & 9,53 & 4,68 & 1 & 28 \\
\hline & BLUECOL & 14,11 & 7,63 & 3 & 48 \\
\hline & F\&MAT & 641,95 & 377,63 & 147,08 & 1983,86 \\
\hline \multirow{2}{*}{$\begin{array}{l}\text { Quasi-fixed } \\
\text { inputs }\end{array}$} & GROOM & 26,18 & 8,77 & 11 & 59 \\
\hline & SROOM & 10,58 & 4,37 & 3 & 31 \\
\hline
\end{tabular}

Table 3 and 4 contains a summary of the scale efficiency measurement results. ${ }^{5}$ It includes the standard DEA results and the test of hypothesis using the homogenous and heterogeneous bootstrap simulations of our model. The number of bootstrap simulation is equal to $2,000(B=2,000)$.

Table 3 presents the results with the schools grouped by size (measured by the number of students). Small schools have less than one thousand students (97 schools), the middle group (129 schools) includes schools with a number of students between 1,000 and 1,500 and the last group includes the large schools, that is, the schools with more than 1,500 students (106 schools). ${ }^{6}$ The total number of schools is 332 . Except for the first line, each cell contains a triple. The three entries are respectively the number of schools that exhibits increasing returns to scale, constant returns to scale and decreasing returns to scale.

\footnotetext{
${ }_{6}^{5}$ Full results are in an Appendix available from the authors upon request.

${ }^{6}$ There is no formal definition of "small", "medium" and "large" institutions in the literature. The corresponding sizes change considerably from one study to the other (see Stiefel et al. (2009) for a complete survey of the problem). We have kept in this paper a breakdown of the schools sizes such that the distribution is relatively homogenous between groups.
} 
Table 3: Results of the test for the homogenous and the heterogeneous bootstrap

\begin{tabular}{|c|c|c|c|c|}
\hline & \multicolumn{3}{|c|}{ School Size } & \multirow{2}{*}{ Total } \\
\hline & Small & Medium & Large & \\
\hline Number of schools & 97 & 129 & 106 & 332 \\
\hline $\begin{array}{c}\text { Scale Efficiency: } \\
\text { Number of schools per } \\
\text { RTS type } \\
(\text { irs }, c r s, d r s)\end{array}$ & $(85,11,1)$ & $(86,23,20)$ & $(26,13,67)$ & $(197,47,88)$ \\
\hline \multicolumn{5}{|l|}{ Scale Efficiency Test: } \\
\hline \multicolumn{5}{|c|}{ 1) Homogenous Bootstrap: } \\
\hline$\alpha=1 \%$ & $(20,77,0)$ & $(6,123,0)$ & $(0,106,0)$ & $(26,306,0)$ \\
\hline$\alpha=5 \%$ & $(35,62,0)$ & $(14,115,0)$ & $(0,105,1)$ & $(49,282,1)$ \\
\hline$\alpha=10 \%$ & $(46,51,0)$ & $(20,109,0)$ & $(0,101,5)$ & $(66,261,5)$ \\
\hline \multicolumn{5}{|c|}{ 2) Heterogeneous Bootstrap } \\
\hline$\alpha=1 \%$ & $(33,64,0)$ & $(0,129,0)$ & $(0,106,0)$ & $(33,299,0)$ \\
\hline$\alpha=5 \%$ & $(10,87,0)$ & $(0,129,0)$ & $(0,106,0)$ & $(10,322,0)$ \\
\hline$\alpha=10 \%$ & $(19,78,0)$ & $(0,129,0)$ & $(0,106,0)$ & $(19,313,0)$ \\
\hline
\end{tabular}

We first present the traditional scale efficiency scores proposed by Färe et al. (1994). The results are grouped by school sizes and each triple contains the number of schools belonging to each category of returns to scale. Clearly, most DMUs are not scale efficient (more than 85\%). More than half of the schools exhibit increasing returns to scale, this result being concentrated in the small and medium size schools ( 85 small and 86 medium size high school). The majority of the large schools (67 out of 106) exhibit decreasing returns to scale. There are fourteen percent of the high schools that exhibit constant returns to scale. These schools are deemed scale efficient. Half of these schools are medium size institutions. The other half is spread evenly between the small and large schools. Clearly, the first analysis using the standard approach does not provide any indication on the optimal size for a school. Therefore, we have to look for an answer somewhere else in order to get guidance in choosing the optimal size. A potential direction to look for is suggested by the fact that these results on the optimal size are based on the raw scores and do not take into account potential sampling variations.

Such a traditional presentation of the results does not include the possibility that the scale efficiency scores might be estimators of a parameter. As explained above, the scores are not measured with respect to the true frontier, but with respect to an estimated frontier. Consequently, the scale efficiency scores are themselves estimates of the true scores, thus subsumed to statistical regularities; hence the results reported above must be accompanied by an estimate of their 
precision. In other words, reported cases of scale inefficiencies in the traditional approach might be the result of sampling variations.

The bootstrap general simulations results are displayed in last rows of Table 3, while Table 4 presents some specific cases. The simulations results are always reported for three different test sizes: $\alpha=1 \%, \alpha=5 \%$ and $\alpha=10 \%$. The application of the test procedure we developed in the previous sections confirms the intuition that sampling variations play a significant role in the estimation of scale efficiencies. The bottom part of Table 3 confirms that the vast majority of the schools operate under constant returns to scale. That is, more than $78 \%$ of the institutions are now deemed scale efficient at all test sizes for both simulation methods, in strong contrast with what we found initially. In other words, the scale inefficiencies identified by the brute scores are spoiled by some noise and most of the decision units are in fact scale efficient. These results are not sensitive to the type of bootstrap procedure, homogenous or heterogeneous, so even the simplest statistical procedure raises some concerns on the interpretation of the straight scores of scale efficiencies without its statistical content. The results in Table 3 allow us to show the existence of a significant relationship between high school sizes and scale efficiency scores. The highest concentration of scale-efficient schools is found among the medium or large size institutions. At test size $\alpha=5 \%$, out of the 282 schools deemed scale efficient using the homogenous bootstrap method, 115 are medium size high schools and 105 are large institutions. For the same test size, the results using the heterogeneous bootstrap simulations lead us to a fairly similar conclusion (out of 322 scale efficient institutions we have 122 medium and 106 large institutions). This also raises the point that when a school is small it is more likely to be scale-inefficient. This casts some doubts on the policy promoted by the department of education that organized a systematic reduction of the school sizes. In lights of our results, this movement is not totally optimal, as in general smaller schools are more likely to have difficulties to be scale efficient than medium and large size institutions.

Table 4 presents the result for specific units. Our approach has focused on unit specific test procedure by contrast to Simar and Wilson (2002) that consider a global test. It compares directly the simulation results obtained using the homogenous and heterogeneous bootstrap to the standard results (ignoring the statistical content of the analysis). Some specific examples of DMUs are presented in the Table and serve to illustrate some general trends. 


\section{[INSERT TABLE 4 HERE]}

The homogenous bootstrap results confirm our intuition as it is impossible to reject the hypothesis of scale efficiency (crs) at a size equal to 5\% for 282 high schools (more than $84 \%$ of the sample). Most of those schools are of either medium or large size (roughly $80 \%$ of the scale efficient schools). There are 49 high schools (more than $14 \%$ of the sample) that exhibit increasing returns to scale. Most of these institutions are small schools. That is, 35 high schools (70\% of the group) have less than one thousand students. High school L1115, with an enrolment of 1801 students, is the only case of decreasing returns to scale (significant at 5\%) and not surprisingly it is a large school. These results are robust to the size. At size $\alpha=10 \%$, we continue to observe a fairly large proportion of high schools, mostly medium and large institutions, that exhibits constant returns to scale (more than $78 \%$ of the sample). There is also a smaller proportion of the high schools (about 20\%), mostly of a small size, that exhibit increasing returns to scale. There are only at most five high schools which can be deemed to operate under decreasing returns to scale and they are all large institutions.

In theory, the heterogeneous bootstrap simulations would provide results closer to the reality of the institutions because they account for the specificities of the production process. The cost for this is important as the procedure is quite involved. The comparison of its performance with the homogenous procedure is thus interesting. The last columns of Table 4 present specific cases for this procedure and must help to understand what is going on in both procedures. A striking point is that the results are very similar to those of the homogenous bootstrap, something we interpret as a confirmation of the robustness of the test procedures and their results. The number of scale efficient DMU increases to $90 \%$ for all three test sizes. In both simulations, most of the DMU exhibit the same type of returns to scale. The heterogeneous bootstrap tends to ascribe the scale efficient label to schools more often than the homogenous procedure, however. None of the high schools exhibit decreasing returns to scale at all three sizes for the heterogeneous bootstrap. One striking feature of these results is the relationship between the types of returns to scale and the enrolment, our size indicator of the schools. The heterogeneous bootstrap simulations are clear on that point: schools of medium and large sizes are all scale efficient. That is, they are deemed to have an optimal size at $\alpha$ equal to $1 \%, 5 \%$ and $10 \%$. The 
DMU that are not efficient, for which the null hypothesis is rejected at $\alpha$ equal to $1 \%$ in the first test, are all schools with less than one thousand students, the smallest schools of our sample.

This result is in line with the conclusion we reached for the homogenous bootstrap simulations. It seems that most medium and large schools are scale efficient, while their smaller counterparts are not. The latter exhibit increasing returns to scale and from the inverse relationship identified between returns to scale and size measured by the number of students, we would be inclined to believe that these schools are too small. Clearly, these conclusions are not good for the policy promoted by the Tunisian department of education. It seems that the route to improve the efficiency of the education system in Tunisia does not go through a systematic reduction of the size of the high schools. To the contrary, the smallest institutions tend to boast bad results in terms of scale efficiency. Some small schools seem to sit on potential performance gains than cannot be exploited due to their size.

\section{Conclusion}

A very entrenched claim by program designers and education system decision makers is that small establishments are better than bigger one for the students (Schneider et al. (2007) for a discussion of this point). Despite the pedagogical arguments to that effect, for economists this problem is directly related to the scale efficiency. In other words, what is the school optimal size so that all resources devoted to the institution are optimally used to produce well educated students? To respond to this question and orient correctly the education policy we need a formal way to determine the optimal size of given educational institutions.

In this article we have developed a procedure to test non parametric statistical hypothesis concerning the scale efficiency of organization, readily implementable to schools. Because there is no asymptotic distribution for the test statistic under the null hypothesis, we used a smooth bootstrap methodology to approximate it. This approach allows us to estimate the $p$-values of the test and to determine a decision rule to accept or reject the null hypothesis. The results of our application of this methodology to the Tunisian secondary schools show on the one hand that scale efficiencies are strongly sensitive to sampling variation and on the other hand that the claim made by the decision makers in the Department of Education in Tunisia that the country should aimed at reducing as much as possible the size of the schools is not verified. Smaller schools are not better and in fact we have shown that they are probably the only one that are systematically 
not of the right size as they are exhibiting increasing returns to scale. It is in medium and large institutions that we can find high schools fully benefiting from scale efficiencies.

We have ignored the socio-economic characteristics of students and the localization of the schools, however. This may eventually force us to qualify our results as rural schools may not be able to increase the number of students because of weak population density for instance. Nonetheless, we do not expect these considerations to change radically the picture we drew of the school efficiency in Tunisia.

\section{References}

Banker, R.D. (1984), "Estimating Most Productive Scale Size Using Data Envelopment Analysis", European Journal of Operational Research 17, 35-44.

Banker, R.D. and Morey, R.C. (1986), "Efficiency Analysis for Exogenously Fixed Inputs and Outputs", Operations Research 34, 513-521.

Banker, R.D. and Thrall, R.M. (1992), "Estimation of Returns to Scale Using Data Envelopment Analysis", European Journal of Operational Research 62, 74-84.

Banker, R.D., Chang, H. and Cooper, W.W. (1994), "Equivalence of Alternative Methods for Returns-to-Scale Estimation in Data Envelopment Analysis", Unpublished working paper.

Banker, R.D., Charnes, A. and Cooper, W.W. (1984), "Some Models for Estimating Technical and Scale Inefficiencies in Data Envelopment Analysis", Management Science 30, 10781092.

Chabotar, K. J. (1989), "Measuring the Costs of Magnet Schools", Economics of Education Review 8, 169-183.

Colegrave, A. D. and Giles, M. J. (2005), "School Cost Functions: A Meta Regression Analysis", Center for Labor Market Research, University of Western Australia Crawley, WA, paper.

Coladarci, T. and Cobb, C. D. (1996), "Extracurricular Participation, School Size, and Achievement and Self-esteem among High School Students: A National Look", Journal of Research in Rural Education 12, 92-103.

Essid, H., Ouellette, P. and Vigeant, S. (2010a), "Measuring Efficiency of Tunisian Schools in the Presence of Quasi-fixed Inputs: A Bootstrap DEA Approach", Economics of Education Review 29, 589-596.

Essid, H., Ouellette, P. and Vigeant, S. (2010b), "Scale Efficiency Tests and DEA Estimation: A Bootstrap Approach ", mimeo, USTL. 
Essid, H., Ouellette, P. and Vigeant, S. (2010c), "A Note on the Convergence of Nonparametric DEA Estimators for Production Efficiency Scores with Quasi-Fixed Inputs", mimeo, Institut des HEC de Carthage.

Farrell, M.J. (1957), "The Measurement of Productive Efficiency", J.Roy.Stat.Soc. A 120, 253290.

Foreman-Peck, J. and Foreman-Peck, L. (2006), "Should Schools Be Smaller ? The SizePerformance Relationship for Welsh Schools", Economics of Education Review 25, 157 171.

Fox, W. F. (1981), "Reviewing Economies of Size in Education", Journal of Education Finance 6, 273-296.

Färe, R. (1988), "Fundamentals of Production Theory", Lecture Notes in Economics and Mathematical Systems, Vol. 311, Springer-Verlag, Berlin Heidelberg.

Färe, R. and Grosskopf, S., (1994), "Estimation of Returns to Scale Using Data Envelopment Analysis: A Comment", European Journal of Operational Research 79, 379-382.

Färe, R. and Grosskopf, S. (1985), "A Nonparametric Cost Approach to Scale Efficiency", Scandinavian Journal of Economics 87, 594-604.

Färe, R., Grosskopf, S. and Lovell C.A.K. (1994), "Production Frontiers", Cambridge University Press, Cambridge.

Kneip, A., Park, B.U. and Simar, L. (1998), "A Note on the Convergence of Nonparametric DEA Estimators for Production Efficiency Scores", Econometric Theory 14, 783-793.

Kumar, R. C. (1983), "Economies of Scale in School Operation: Evidence from Canada", Applied Economics 15, 323-340.

Kuziemko, I. (2006), "Using Shocks to School Enrollment to Estimate the Effect of School Size on Student Achievement", Economics of Education Review 25, 63-75.

Leithwood K. and Jantzi D. (2009), "A Review of Empirical Evidence about School Size Effects: A Policy Perspective", Review of Educational Research 79, 464-490.

McGuire, K. (1989), "School Size: The Continuing Controversy" Education and Urban Society 21, 164-174.

Monk, D. H. (1987), "Secondary School Size and Curriculum Comprehensiveness", Economics of Education Review 6, 137-150. 
Morris, H. J. (1964), Relationship of School Size to Per Pupil Expenditures in Secondary Schools in the Southern Region, Unpublished doctoral dissertation, George Peabody College for Teachers, Nashville, TN.

Park, B. U., Jeong, S. -O. and Simar, L. (2010), "Asymptotic Distribution of Conical-Hull estimators of Directional Edges", The Annals of Statistics 38, No. 3, 1320-1340.

Schneider, B., Wyse, A. E., and Keesler, V. (2007), "Is Small Really Better ? Testing Some Assumptions about High School Size", In L. Tom \& F.M. Hess (Eds.), Brookings Papers on Education Policy 2006/2007, Washington, DC: The Brookings Institution.

Schuster, E.F. (1985), "Incorporating Support Constraints into Nonparametric Estimators of Densities", Communications in Statistics-Theory and methods 14, 1123-1136.

Silverman, B.W. (1986), "Density Estimation for Statistics and Data Analysis", Chapman and Hall.

Simar, L. and Wilson, P.W. (2002), "Non-Parametric Tests of Returns to Scale", European Journal of Operational Research 139, 115-132.

Simar, L. and Wilson, P.W. (2000), "A General Methodology for Bootstrapping in nonparametric frontier models", Journal of Applied Statistics 27, 779-802.

Simar, L. and Wilson, P.W. (1998), "Sensitivity Analysis of Efficiency Scores: How to Bootstrap in Nonparametric Frontier Models", Management Science 44, 49-61.

Stiefel, L., Schwartz, A.E., Iatarola, P. and Chellman, C.C. (2009), "Mission Matters: The Cost of Small High Schools Revisited", Economics of Education Review 28, 585-599.

Turner, C. C., and Thrasher, J. M. (1970), "School Size Does Make a Difference", San Diego, CA: Institute for Educational Management (ERIC Document Reproduction Service No. ED 043946).

Word Bank, (2008), The Road Not Traveled Education Reform in the Middle East and North Africa. The World Bank, Washington, D.C. 
Table 4: Homogenous and the heterogeneous bootstrap test results: some specific cases

$$
\begin{array}{cccc}
\text { DMU Enrolment } & \hat{S}_{1 j} & \text { Initial } \\
& & & \begin{array}{c}
\text { Scale } \\
\text { efficiency }
\end{array}
\end{array}
$$
Homogenous bootstrap simulation results$$
\hat{p}_{j}
$$

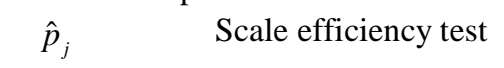

\begin{tabular}{cccc}
$\hat{p}_{j}$ & \multicolumn{3}{c}{ Scale efficiency test } \\
$($ Test 2) & & & \\
& $\alpha=0,01$ & $\alpha=0,05$ & $\alpha=0,1$ \\
0,2855 & & & \\
0,4490 & $c r s$ & $c r s$ & $c r s$ \\
0,9555 & $c r s$ & $c r s$ & $c r s$ \\
0,9695 & $c r s$ & $c r s$ & $c r s$ \\
0,9045 & $c r s$ & $d r s$ & $d r s$ \\
0,9690 & $c r s$ & $c r s$ & $c r s$ \\
0,5420 & $c r s$ & $c r s$ & $c r s$ \\
0,9925 & $c r s$ & $c r s$ & $c r s$ \\
0,0005 & $i r s$ & $i r s$ & $i r s$ \\
0,0090 & $i r s$ & $i r s$ & $i r s$ \\
0,9740 & $c r s$ & $c r s$ & $d r s$ \\
0,0115 & $c r s$ & $i r s$ & $i r s$ \\
0,8920 & $c r s$ & $c r s$ & $d r s$ \\
0,1995 & $c r s$ & $c r s$ & $d r s$ \\
0,8880 & $c r s$ & $c r s$ & $d r s$
\end{tabular}

Heterogeneous bootstrap simulation results

\begin{tabular}{cccc}
$\hat{p}_{j}$ & $\hat{p}_{j}$ & \multicolumn{2}{c}{ Scale efficiency test } \\
$($ Test 1$)$ & $($ Test 2$)$ & $\alpha=0,01 \quad$ & $\alpha=0,05 \quad \alpha=0,1$
\end{tabular}

$\begin{array}{lllll}0,8305 & 0,5325 & \text { crs } & \text { crs } & \text { crs } \\ 0,6195 & 0,4725 & \text { crs } & \text { crs } & c r s \\ 0,5790 & 0,9450 & c r s & c r s & c r s \\ 0,9075 & 0,9405 & c r s & c r s & c r s \\ 0,4040 & 0,9480 & c r s & c r s & c r s \\ 0,9850 & 0,9515 & c r s & c r s & c r s \\ 0,3620 & 0,3280 & c r s & c r s & c r s \\ 0,9845 & 0,9915 & c r s & c r s & c r s \\ 0,1480 & 0,1455 & c r s & c r s & c r s \\ 0,1085 & 0,1060 & c r s & c r s & c r s \\ 0,7040 & 0,9495 & c r s & c r s & c r s \\ 0,3475 & 0,3020 & c r s & c r s & c r s \\ 0,5105 & 0,9500 & c r s & c r s & c r s \\ 0,6710 & 0,6060 & c r s & c r s & c r s \\ 0,3940 & 0,9405 & c r s & c r s & c r s\end{array}$

